COMMENT. MRIs repeated at age 10 months may disclose delayed myelination in infants with WS. The delayed myelination is not always explained by ACTH therapy and may reflect the organic brain lesion causing the seizures.

\title{
FRAGILE X MUTATIONS AND EPILEPSY
}

A posiible link between predisposition for epilepsy and mutations in the fragile $X$ mental retardation-1 gene (FMR) was investigated in the Neuropediatric Department, Behandlungszentrum Vogtareuth; and Laboratory of Genetic Diagnostics, Munchen, Germany. EEGs performed on 14 patients with an amplification in the FMR-1 gene showed focal sharp waves and partial seizures in sleep in 8 boys, aged 4-8 years. Of 16 children with rolandic epilepsy (BECT) studied for FMR-1 gene mutations, 1 boy was positive. (Kluger G, Bohm I, Laub MC, Waldenmaier C. Epilepsy and fragile X gene mutations. Pediatr Neurol Nov 1996;15:358-360). (Respond: Dr Kluger,Neuropediatric Department, Behandlungszentrum Vogtareuth, Krankenhausstrabe 20, D-83569 Vogtareuth, Germany).

COMMENT. A higher incidence of seizures or EEG abnormalities may be expected in boys with fragile $\mathrm{X}-1$ gene mutations.

\section{ANTIEPILEPTIC DRUGS}

\section{PHENYTOIN AND CARBAMAZEPINE TERATOGENICITY}

In a prospective, controlled, and blinded study of 36 mother-child pairs exposed to carbamazepine (CBZ) monotherapy, 34 pairs exposed to phenytoin (DPH) monotherapy, and 9 nonmedicated epileptic women and their children, the patterns of malformations in the children exposed to potential teratogenic factors were compared with matched mother-child pairs exposed to nonteratogens, at the Hospital for Sick Children, Toronto, Canada. There was no correlation between the daily dose of DPH or CBZ and number of malformations. Microcephaly occurred in $6 \%$ of children exposed to DPH and $8.8 \%$ of those exposed to CBZ, but not in medicated nonepileptic or nonmedicated epileptic subgroups. Malformations in $8.8 \%$ of DPH and $5.7 \%$ of CBZ exposed children were not significantly different from controls. Minor anomalies in children exposed to either AED were more frequent than in controls, with a relative risk of 2.1. Hypertelorism was more frequent among DPH-exposed offspring; $25 \%$ incidence vs $11 \%$ in controls. High forehead, frontal bossing, malar hypoplasia, epicanthus and micrognathia occurred in association with untreated epilepsy, as well as DPH and CBZ treatment. (Nulman I, Scolnik D, Chitayat D, Farkas LD, Koren G. Findings in children exposed in utero to phenytoin and carbamazepine monotherapy: independent effects of epilepsy and medications. Am I Med Genet Jan 1997;68:18-24). (Respond: Gideon Koren MD, The Hospital for Sick Children, 555 University Avenue, Toronto, Ontario, M5G $1 \mathrm{X8}$, Canada).

COMMENT. AEDs and epilepsy have teratogenic effects that are independent and result in minor anomalies in infants exposed in utero. Previous studies have shown that valproate and carbamazepine are associated predominantly with spina bifida and hypospadias, whereas barbiturates and phenytoin may induce congenital heart malformations and facial clefts. None of the AEDs is free of possible adverse effects on the fetus. Experience with 
gabapentin, lamotrogine, and other newer agents during pregnancy is relatively sparse, but teratogenicity has not been reported.

\section{GROWTH SUPPRESSION WITH ACETAZOLAMIDE}

The effect of acetazolamide on growth of children with epilepsy was evaluated at Osaka Medical Center, Japan. Standard scores of height and weight were compared in 17 children receiving acetazolamide as an adjunct to AED monotherapy: 1) before AED treatment; 2) during AED monotherapy; 3) during adjunct acetazolamide therapy; and 4) after acetazolamide had been discontinued. Both height and weight were significantly reduced during acetazolamide administration, and growth returned to the original level after acetazolamide was withdrawn. The degree of growth suppression was not related to the age, duration, dosage, or the concomitant AED therapy. Metabolic acidosis induced by acetazolamide was postulated as the cause. (Futagi Y, Otani $\mathrm{K}$, Abe J. Growth suppression in children receiving acetazolamide with antiepileptic drugs. Pediatr Neurol Nov 1996;15:323-326). (Respond: Dr Futagi, Division of Pediatric Neurology, Osaka Medical Center and Research Institute, 840 Murodo-cho, Izumi, Osaka 590-02, Japan).

COMMENT: Anorexia and loss of weight have been reported with acetazolamide therapy for epilepsy (Millichap et al. 1956, 1964), but this seems to be the first report of acetazolamide-induced growth suppression. Height and weight should be monitored carefully during acetazolamide therapy.

Since the metabolic side effects of the ketogenic diet are similar to those of acetazolamide (Millichap JG. Progress in Pediatric Neurology I, PNB Publ, 1991:pp85-88), and loss of weight is a common occurrence with the initiation of the diet, monitoring of height and weight are equally important during ketogenic dietary therapy. Two children under one year of age showed no increase in weight, length or head circumference during a six month period on the diet (Schwartz RH et al, 1989), and the monitoring of acetazolamide or the ketogenic diet in infants and young children should be especially strict.

\section{ATTENTION DEFICIT DISORDERS}

\section{ADHD AND PSYCHOACTIVE SUBSTANCE ABUSE}

The effect of attention-deficit hyperactivity disorder (ADHD) compared to psychiatric comorbidity, familiality, and adversity, as risk factors for psychoactive substance use disorder (PSUD) was evaluated at the Department of Psychiatry, Massachusetts General Hospital, Boston, MA. Using baseline and 4year follow-up data from 140 ADHD and 120 normal control subjects, the rates of alcohol or drug abuse or dependence (PSUD) were $15 \%$, with no differences between groups. Conduct and bipolar disorders were predictive of PSUD, but these associations were independent of ADHD. Oppositional defiant disorder, uncomplicated by conduct disorder, did not predict PSUD. Family history of substance dependence and antisocial disorders was associated with PSUD in controls but less so in ADHD probands. Family history of ADHD was not associated with risk of PSUD. (Biederman J, Wilens T, Mick E et al. Is ADHD a risk factor for psychoactive substance use disorders? Findings from a fouryear prospective follow-up study. L Am Acad Child Adolesc Psychiatry Jan 1997;36:21-29). (Reprints: Dr Biederman, Pediatric Psychopharmacology Unit (ACC 725), Massachusetts General Hospital, Fruit Street, Boston, MA 02114). 